\title{
Transition Micromagnetic Structures in Asymmetric Vortexlike Domain Walls (Static Solutions and Dynamic Reconstructions)
}

\author{
V. V. Zverev ${ }^{a, *}$ and B. N. Filippov ${ }^{a, b}$ \\ ${ }^{a}$ Ural Federal University, ul. Mira 19, Yekaterinburg, 620002 Russia \\ ${ }^{b}$ Institute of Metal Physics, Ural Branch, Russian Academy of Sciences, ul. S. Kovalevskoi 18, Yekaterinburg, 620990 Russia \\ *e-mail:vvzverev49@gmail.com \\ Received November 9, 2012
}

\begin{abstract}
The possible types of transition structures with a three-dimensional magnetization distribution over regions in the vortex asymmetric domain walls that exist in magnetically uniaxial soft magnetic films with in-plane anisotropy are studied by computer simulation in terms of a micromagnetic approach. It is shown that the possible structure types include both the type of vertical Bloch lines that was discussed earlier in other works and new types, namely, singular (Bloch) points and clusters consisting of vertical Bloch lines and Bloch points. The spatial configurations of the transition structures are calculated and their topological properties are found. The numerical simulation of the dynamics of closely spaced substructure regions reveals various scenarios of their interaction, including annihilation accompanied by energy release and the excitation of nonlinear waves.
\end{abstract}

DOI: $10.1134 / \mathrm{S} 1063776113080219$

\section{INTRODUCTION}

The practically important properties of materials with magnetic ordering (magnetic losses, hysteresis, etc.) are substantially determined by the presence of locally heterogeneous magnetization structures in them and the dynamic properties of these structures. The characteristic sizes of these structures are such that they are large-scale as compared to the interatomic distances in a crystal lattice and are small from a macroscopic viewpoint. This specific feature makes it possible to use a micromagnetic approach, where the energy of a magnet is determined phenomenologically and magnetization is taken to be classical continuum vector field $\mathbf{M}[1,2]$.

The nonlinear equations of the micromagnetic theory can be solved analytically only in a few particular cases. Therefore, researchers have to use numerical procedures to obtain theoretical information on the state and motion of magnetization. At present, magnetization dynamics can be simulated in a threedimensional manner owing to the progress in computer technologies [3]. As a result, the formation of the magnetic properties of real substances can be studied from a rather general standpoint, which is not restricted to simple schematic models.

Stable micromagnetic structures (MSs), the failure of which requires the overcoming of energy barriers, are of practical interest as the basis for creating new data storage and processing technologies. In particular, MSs in which $\mathbf{M}$ distributions cannot be transformed into each other or into a uniform distribution using continuous deformations (topological MSs) are stable. So-called vertical Bloch lines (VBLs), which (by definition) represent transition regions between two parts of Bloch walls, are topological objects. VBLs were first studied in relation to the problem of creating new energy-independent and radiation-resistant magnetic memory devices [4]. At present, it is interesting to analyze the effect of VBLs on the dynamic properties of domain walls in new-type information media [5]. Note that the term VBL was introduced in early works, where simple models of domain walls (in particular, walls with a one-dimensional internal structure) were considered. A three-dimensional simulation showed that the transition regions in domain walls have a complex spatial structure and are not simply lines; therefore, we will call them transition micromagnetic structures (TMSs).

When studying magnetically uniaxial films with inplane anisotropy using permalloy films as a typical example, researchers first used the concept of onedimensional and two-dimensional $\mathbf{M}$ distributions in walls and their substructure elements (Bloch lines), respectively [6]. Only as a result of long-term efforts, it was found (theoretically [7] and experimentally [8]) that the structure of walls in such films has a twodimensional asymmetric $\mathbf{M}$ distribution. When analyzing the experimental data from $[9,10]$, the authors of [9] concluded that three types of TMSs that separate regions of asymmetric domain walls with different structures (with the location of vortices on the left or right of the central line of a wall, with the clock- or counterclockwise rotation of magnetization in vortices) can exist. Three-dimensional numerical simula- 
tion, which was first performed in [11-14], supported the two-dimensionality and asymmetry of the walls and showed that TMSs that separate regions in asymmetric walls are substantially three-dimensional local objects. The authors of those works found the main types of static configurations of TMSs in iron [11, 12] and permalloy $[13,14]$ films, which include various types of VBLs.

We think that the static properties of vortexlike walls with TMSs are still incompletely understood. To establish whether such structures are stable or metastable (similarly to model walls with a one-dimensional $\mathbf{M}$ distribution), we calculate the total energies of various $\mathbf{M}$ configurations. Moreover, based on the simulation of the $\mathbf{M}$ distribution in films $80-140 \mathrm{~nm}$ thick, we will find new types of TMSs, which include singular points (SPs; they are also called Bloch points [6]). The M distribution in the vicinity of SP is such that magnetization takes all possible values on an SPcontaining sphere of an arbitrarily small radius [15]. TMSs can be both single SPs and combined MSs (clusters) containing several SPs and VBLs. The assumption of the presence of SP in the TMSs of the type under study was made in [10]; however, solutions with SPs were not found in [11-14].

Significant attention has recently been devoted to studying vortex MSs. Apart from vortex domain walls (where vortex axes are parallel to the film plane) [1618], researchers considered single vortices (antivortices) with their axes normal to the film surface and systems of interacting vortices (antivortices) of this type. It was found that the high-rate "switching" of vortex polarity is a complex transient process, which is accompanied by the generation and annihilation of vortex-antivortex pairs [19-22] and the emission of nonlinear waves [23-25]. In this work, we will show that the structural transformations of TMS separating regions in asymmetric vortex domain walls can be rapid processes accompanied by annihilation in vortex-antivortex pairs, energy release, and the initiation of wave processes.

We restrict ourselves to simulating the dynamics of transient processes for specially created nonequilibrium initial states in the absence of an external magnetic field. This approach permits us to estimate the degree of stability of various configurations by dividing them into arbitrarily stable (long-lived metastable, with a lifetime of much longer than $1 \mathrm{~ns}$ ) and shortlived configurations. We will also classify the main scenarios of the decay and restructuring of short-lived configurations.

\section{FORMULATION OF THE PROBLEM}

We consider a magnetically uniaxial film with inplane anisotropy and a low quality factor $Q \ll 1(Q=$ $K / 4 \pi M_{s}^{2}$, where $K$ is the anisotropy constant and $M_{s}$ is the saturation magnetization). Let axes $y$ and $z$ be par- allel and axis $x$ be perpendicular to the film surface and axis $z$ be oriented along the easy magnetization axis (EA).

Equilibrium magnetization distributions were obtained by minimizing the total energy of the wall

$$
E=\iiint_{V}\left(w_{e}+w_{a}+w_{m}\right) d \mathbf{r}
$$

where

$$
\begin{aligned}
& w_{e}=\frac{A}{M_{s}^{2}}\left\{\left(\frac{\partial \mathbf{M}}{\partial x}\right)^{2}+\left(\frac{\partial \mathbf{M}}{\partial y}\right)^{2}+\left(\frac{\partial \mathbf{M}}{\partial z}\right)^{2}\right\}, \\
& w_{a}=-\frac{K}{M_{s}^{2}}(\mathbf{k} \cdot \mathbf{M})^{2}, \quad w_{m}=-\frac{1}{2} \mathbf{M} \cdot \mathbf{H}^{(m)}
\end{aligned}
$$

are the exchange, magnetic anisotropy, and magnetostatic energy densities, respectively, expressed in terms of magnetization $\mathbf{M}, A$ is the exchange interaction parameter, and $\mathbf{k}$ is the unit vector directed along axis $z$. Magnetostatic field $\mathbf{H}^{(m)}$ is determined by solving magnetostatic equations with the corresponding boundary conditions. Calculation area $V$ has the shape of a parallelepiped with sizes $L_{x}$ (film thickness), $L_{y}$, and $L_{z}$ along the corresponding axes. The boundary conditions on the film surface $\left(x=0, x=L_{x}\right)$ correspond to unfixed magnetization [2]. At faces $y=0$ and $y=L_{y}$, where matching with domains takes place, we have

$$
\left.\mathbf{M}\right|_{y=0}=-\mathbf{k} M_{s},\left.\quad \mathbf{M}\right|_{y=L_{y}}=\mathbf{k} M_{s} .
$$

In addition, we use the following periodic boundary conditions:

$$
\left.\mathbf{M}\right|_{z=0}=\left.\mathbf{M}\right|_{z=L_{z}}
$$

With these conditions (which are analogous to the Born-Kármán conditions), we can avoid taking into account the effects related to a break in $\mathbf{M}$ at the end faces of a domain wall. When calculating $\mathbf{H}^{(m)}$, we take into account the presence of an infinite chain of regions with the same $\mathbf{M}$ distribution along $z$ (when the contributions from distant regions are summed up, $\mathbf{M}$ is approximated by a one-dimensional continuum distribution to avoid the cutoff of summation [26]).

Figure 1 shows the structure of the asymmetric Bloch domain wall (which is also called a $C$-like wall [11-14]) that separates domains with orientations $\mathbf{M}= \pm \mathbf{k} M_{s}$. The $\mathbf{M}$ distribution on the film surface coincides with the $\mathbf{M}$ distribution in a Néel wall and is vortexlike in the section performed by the plane normal to axis $z$. The visible boundaries of the region $M_{z}(\mathbf{r})>0$ in Fig. 1 are gray. The invisible part of the boundary of this region is represented by the central surface of the wall $M_{z}(\mathbf{r})=0$, which intersects plane $x y$ along curve $\sigma$ (neighboring curves are determined by equations $M_{z}= \pm 0.3 M_{s}$ ). The vortex axis in Fig. 1 is on the left of the center of the wall (left-hand wall). 


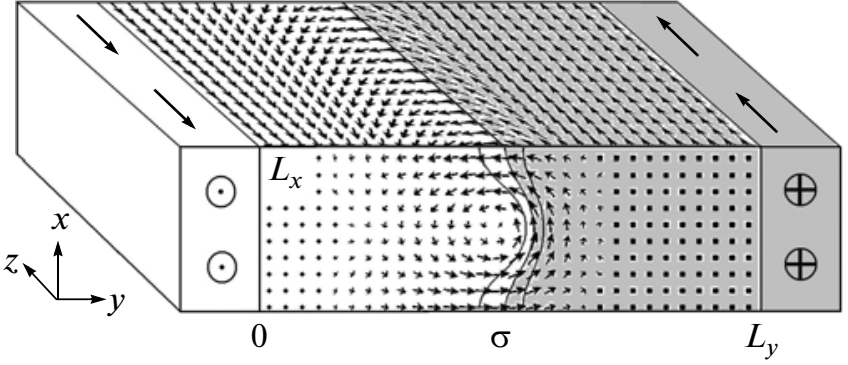

Fig. 1. Spatial distribution of magnetization in a vortex asymmetric domain wall.

Note that this $\mathbf{M}$ distribution makes it possible to close the magnetic flux inside the sample almost completely and to decrease the magnetostatic energy. In addition, there are no regions with strongly heterogeneous $\mathbf{M}$, so that the exchange energy is rather low.

By making rotation through $\pi$ about the abscissa axis, we transform the domain wall shown in Fig. 1 into a right-hand wall with the opposite magnetization rotation direction in the vortex. Another transformation (inversion $\mathbf{M} \longrightarrow-\mathbf{M}$ ) and the subsequent mirror reflection relative to plane $x y$ only change the direction of $\mathbf{M}$ rotation in the vortex and retain a left-hand character of the wall. By combining these transformations, we can convert a left-hand wall into a right-hand wall without changing the direction of $\mathbf{M}$ rotation. Thus, there are four configurations of a wall connected by symmetry transformations. In the absence of an external magnetic field, the energies of these configurations are the same (fourfold degeneracy). Due to the history of the system or the effect of some external factors, a domain wall can consist of regions having different configurations and separated by TMSs. Since a region with a certain configuration can be adjacent to a region having one of the remaining three configurations, there are at least three types of TMSs [11-14].

To obtain TMSs of given types as a result of minimizing energy (1), we use specially chosen initial con- ditions. The process of searching for solutions consists of two stages and is schematically shown in Fig. 2. At the first stage, a piecewise constant magnetization distribution is chosen as initial to roughly imitate the $\mathbf{M}$ distribution in an asymmetric domain wall. When minimizing the energy, we find a three-dimensional distribution that is translation-invariant relative to the $z$ axis and corresponds to a left-hand domain wall. At the second stage, we transform the obtained distribution by one of the three methods described above in field $\Omega$ located between planes $\Lambda_{\mathrm{I}}$ and $\Lambda_{\mathrm{II}}$ and repeat energy minimization. The $\mathbf{M}$ configuration in the plane $z=0$ (i.e., on the visible end face of the sample) corresponds to an untransformed left-hand wall. The $\mathbf{M}$ configurations in the sections $z=L_{z} / 2$ in field $\Omega$ that result from these transformations are shown in the left panel in Fig. 2.

After these transformations, the directions of $\mathbf{M}$ vectors on different sides of each of the $\Lambda_{\mathrm{I}}$ and $\Lambda_{\text {II }}$ planes either coincide or are opposite in the middle part of the sample $\left(y \approx L_{y} / 2\right)$ on the film surface $x=0$ and $x=L_{x}$. As shown in [11-14], vortex and antivortex structures form at the boundary surfaces in the last case; in particular, these structures on the surface $x=$ $L_{x}$ are located near points $v$ and $\bar{V}$ in Fig. 2. Near the centers of the vortex and antivortex, there are regions where $\left|M_{x}\right| \sim M_{s}$ (we will call them magnetostatic poles using this term here in this narrow meaning). TMSs with different $\mathbf{M}$ directions in magnetostatic poles have different internal structures. To form TMSs with given $\mathbf{M}$ directions in poles, it is sufficient to introduce thin layers in which $M_{x}$ is equal to $M_{s}$ or $-M_{s}$ throughout the layer or $M_{x}= \pm M_{s}$ at $0<x<L_{x} / 2$ and $M_{x}=\mp M_{s}$ at $L_{x} / 2<x<L_{x}$ along planes $\Lambda_{\mathrm{I}}$ and $\Lambda_{\text {II }}$ during the formation of initial $\mathbf{M}$ distributions before the second energy minimization (three versions of $\mathbf{M}$ distributions in such layers are shown in the right panel in Fig. 2).

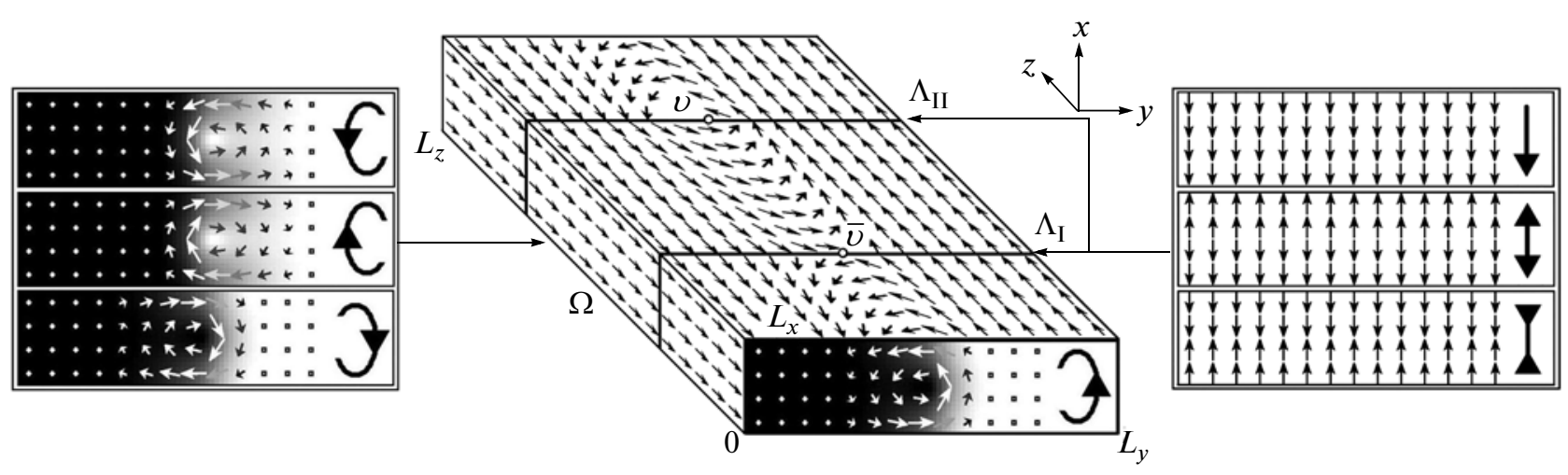

Fig. 2. Schematic diagram for constructing initial distributions for the calculation of static $\mathbf{M}$ configurations. In the sections normal to axis $z$, the color intensity is proportional to $M_{z}$. The pictograms in the form of open ovals with arrows and vertical arrows are intended to identify magnetization distributions, being their formalized images. 


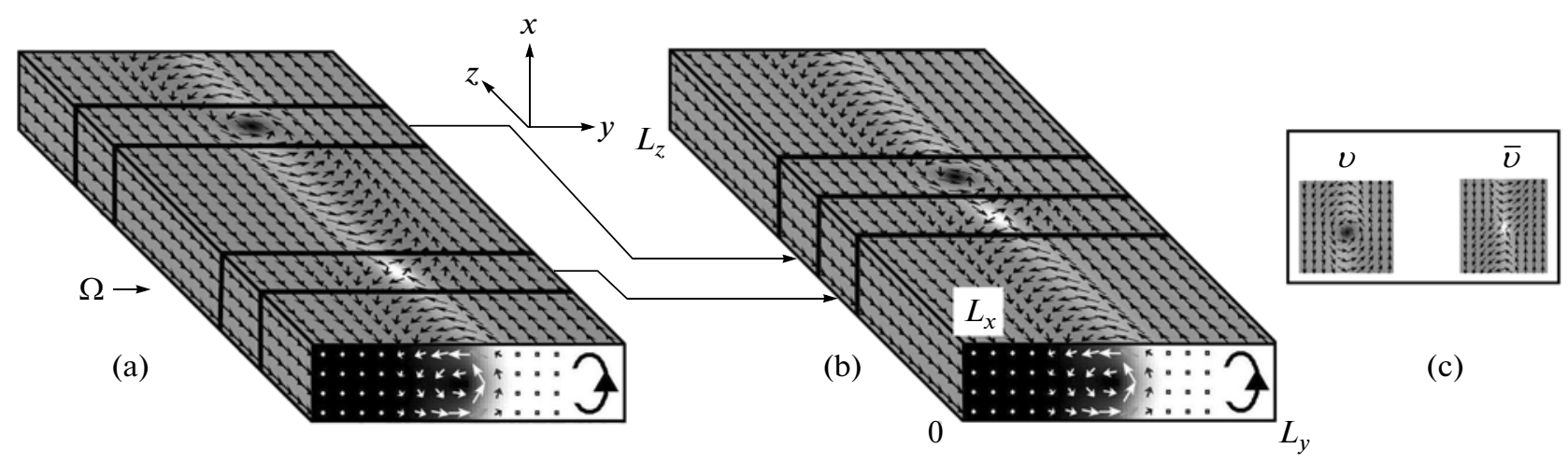

Fig. 3. Schematic diagram for constructing nonequilibrium initial magnetization distributions to simulate dynamic processes.

To describe the magnetization dynamics, we perform a numerical solution of the Landau-LifshitzHilbert equation

$$
\frac{d \mathbf{M}}{d t}=-|\gamma|\left[\mathbf{M} \times \mathbf{H}_{\mathrm{eff}}\right]+\frac{\alpha}{M_{s}}\left[\mathbf{M} \times \frac{d \mathbf{M}}{d t}\right] .
$$

Here, $\gamma$ is the gyromagnetic ratio; $\alpha$ is the damping parameter; and $\mathbf{H}_{\mathrm{eff}}=\mathbf{H}_{e}+\mathbf{H}_{a}+\mathbf{H}^{(m)}$ is the effective field consisting of exchange field $\mathbf{H}_{e}=2 A M_{s}^{2} \Delta \mathbf{M}$, magnetic anisotropy field $\mathbf{H}_{a}=2 K M_{s}^{-2} \mathbf{k}(\mathbf{k} \cdot \mathbf{M})$, and magnetostatic field $\mathbf{H}^{(m)}$.

To obtain the $\mathbf{M}$ distributions that generate nontrivial sequences of fast dynamic transformations, we performed artificial restructuring of the static distributions preliminarily found by energy minimization. Let us dwell on this problem. In Fig. 3a, the $\mathbf{M}$ distribution in the sample corresponds to one of the solutions found by minimizing Eq. (1) (hereafter, the gray density in the flat sample boundary is proportional to the projection of vector $\mathbf{M}$ onto the coordinate axis normal to this boundary: the larger the projection, the lighter the color). The nonequilibrium configurations used as initial ones to study the dynamics are formed as a result of the approach of TMS-containing regions using a piecewise-linear transformation (transition from the configuration shown in Fig. $3 a$ to that in Fig. 3b).

Some TMSs include vortices and antivortices (correspondingly, magnetostatic poles) lying on the film surface. The surface structures of magnetization near vortex $v$ and antivortex $\bar{V}$ are shown in Fig. 3c, where the degree of shading is proportional to $M_{x}$. In this work, we only consider the TMSs with at most one pole in each boundary surface of the film. When such TMSs move toward each other, the magnetization dynamics is substantially determined by the character of vortex-antivortex interaction; in particular, they can annihilate. We will show that this behavior can cause topological transformations of magnetization. To this end, we determine the normalized field of the directions of the projections of $\mathbf{M}$ onto the boundary plane,

$$
\tilde{\mathbf{m}}=\mathbf{M}_{\perp} /\left|\mathbf{M}_{\perp}\right|,
$$

where $\mathbf{M}_{\perp}=\mathbf{M}-\mathbf{i}(\mathbf{i} \cdot \mathbf{M})$ and $\mathbf{i}$ is the unit vector of axis $x$. Two-dimensional vector field $\tilde{\mathbf{m}}$ has singularities at the centers of the vortices (antivortices), where $\mathbf{M}= \pm \mathbf{i} M_{s}$. Following [27, 28], we determine the degree of mapping of the points in plane closed contour $\Gamma=\{\mathbf{r}(t), 0 \leq t<2 \pi\}$ lying on the film surface onto the circle $\tilde{\mathbf{m}}=1$,

$$
j=\frac{1}{2 \pi} \oint d t\left(\tilde{m}_{\bar{y}} \frac{d \tilde{m}_{z}}{d t}-\tilde{m}_{z} \frac{d \tilde{m}_{y}}{d t}\right)
$$

(vector $\tilde{\mathbf{m}}$ components are considered to be taken at the points of contour $\Gamma$ ). The modulus of index $j$ is the number of revolutions executed by $\tilde{\mathbf{m}}$ during single passage along $\Gamma: j>0$ if $\tilde{\mathbf{m}}$ rotates in the sense of the passage of the contour and $j<0$ in the opposite case. If a vortex exists inside the contour, we have $j=1$; if an antivortex exists there, $j=-1$. If a vortex-antivortex pair or no vortex structure exists inside a contour, we have $j=0$. Index $j$ is retained during continuous magnetization distribution deformations; that is, it is a topological invariant [28]. The topological transformations related to a change in $j$ can be called surface transformations, since a vortex and an antivortex in MS appear near the boundary film surfaces.

Let family $\left\{\Gamma(\varsigma), \varsigma \in\left[0, L_{z}\right]\right\}$ is made up of closed contours each of which consists of four directed segments that sequentially connect the points $\left[x_{0}, 0,0\right]$, $\left[x_{0}, L_{y}, 0\right],\left[x_{0}, L_{y}, \varsigma\right]$, and $\left[x_{0}, 0, \varsigma\right]\left(x_{0}=0\right.$ and $L_{x}$ for the lower and upper film boundaries, respectively). When calculating index (6) for various $\Gamma(\varsigma)$ families, we observe a jump of \pm 1 in the $j(\varsigma)$ curve every time when $\varsigma$ is equal to the $z$ coordinate of a vortex (antivortex).

We can find the spatial position and the type of SP of field $\mathbf{M}$ by calculating the values of another topological invariant, which has the meaning of the map- 
Table 1. Configurations of initial distributions (IDs) and the static TMS configurations obtained by energy minimization

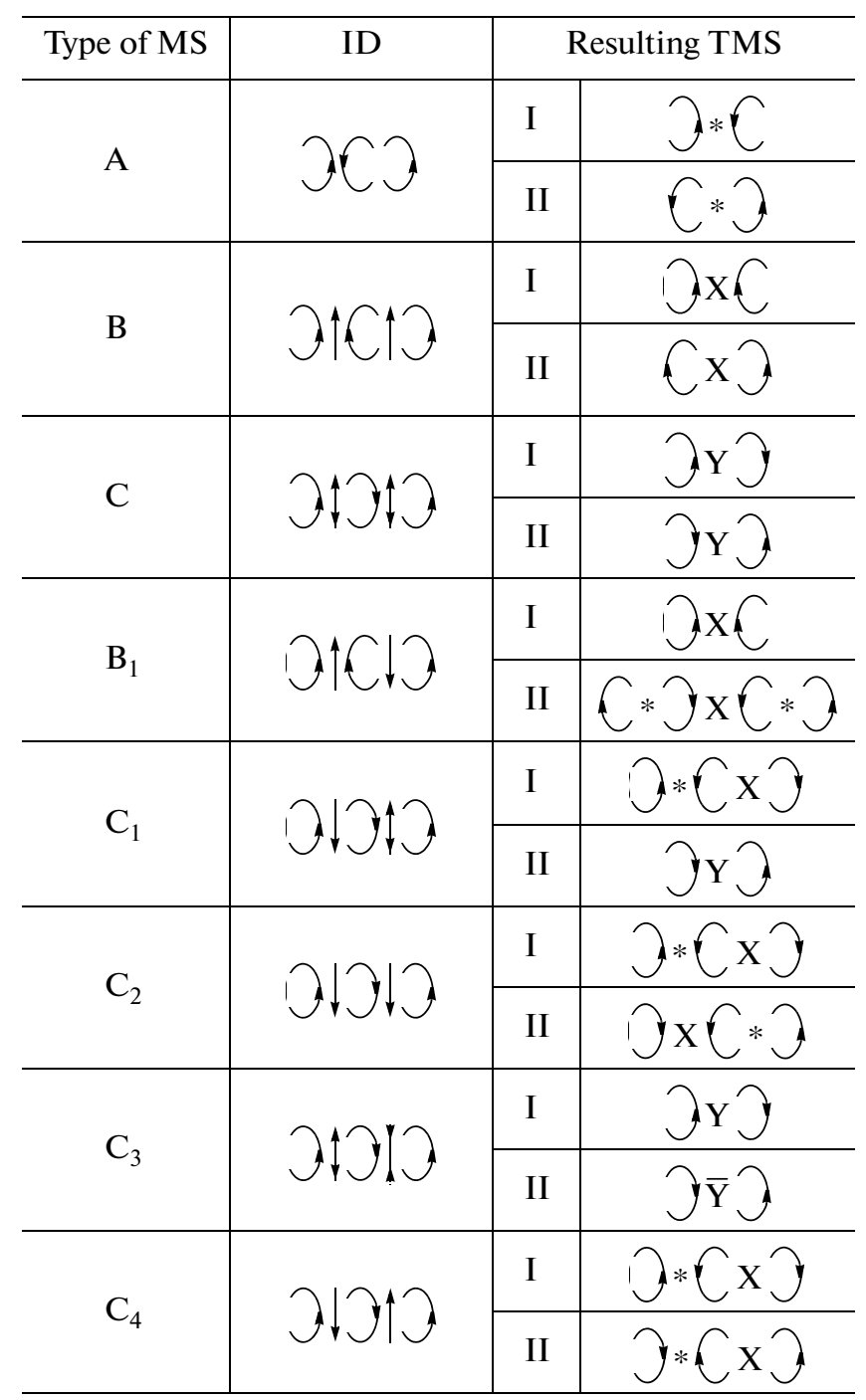

ping of closed surface $S$ in the coordinate space into sphere $|\mathbf{M}|=M_{s}$ in the magnetization vector space. Following [6, 29], we represent this invariant as the surface integral of the gyrotropic vector $\mathbf{g}$ density expressed in terms of $\mathbf{m}=\mathbf{M} / M_{s}$,

$$
\begin{gathered}
\chi=\frac{1}{4 \pi} \oint_{S} \mathbf{g}(\mathbf{r}) d \mathbf{s}, \\
\mathbf{g}(\mathbf{r})=\frac{1}{2} \sum_{i j k} \varepsilon_{i j k} m_{i}\left[\nabla m_{j}, \nabla m_{k}\right] \\
=\frac{1}{m_{\gamma}}\left[\nabla m_{\alpha}, \nabla m_{\beta}\right] .
\end{gathered}
$$

Here, $\varepsilon_{i j k}$ is the Levi-Civita symbol, and one of the substitutions $(\alpha, \beta, \gamma)=(x, y, z),(y, z, x),(z, x, y)$ is assumed to take place. Equivalent definitions of invariant $\chi$ are given in [27, 28, 30, 31]. We call the topological transformations changing $\chi$ solid transformations, since they are related to the appearance and disappearance of SPs, which are three-dimensional objects, and can occur far from the film boundary. Note that the authors of [31], where the states of a two-dimensional ferromagnet with homogeneous magnetization at infinity were considered, expressed mapping (7) through an integral over a plane with identified infinitely distant points.

We now form a family of closed surfaces $\{S(\varsigma), \varsigma \in$ $\left.\left[0, L_{z}\right]\right\}$ consisting of the boundary surfaces of parallelepipeds

$V(\varsigma)=\left((x, y, z), 0<x<L_{x}, \quad 0<y<L_{y}, \quad 0<z<\varsigma\right)$.

Having a sample with one or several SPs and calculating quantities (7) numerically, we detect a jump of \pm 1 in the $\chi(\varsigma)$ curve at every coincidence of $\varsigma$ with the $z$ coordinate of SP.

The magnetization distributions that do not contain TMS and contain TMSs with various numbers of SPs, vortices, and antivortices are topologically pairwise nonequivalent. In particular, this fact is reflected in different forms of functions $j(\varsigma)$ and $\chi(\varsigma)$. We will show that both surface (annihilation of vortex-antivortex pairs changing invariant $j$ ) and solid (generation of SPs at the film boundary and annihilation of SPs at internal points; invariant $\chi$ changes) topological transformations are possible in a sample with finite sizes.

The numerical calculations were performed using the parameters characteristic of permalloy films with a zero-magnetostriction composition: $A=10^{-11} \mathrm{~J} / \mathrm{m}$, $K=10^{2} \mathrm{~J} / \mathrm{m}^{3}$, and $M_{\mathrm{s}}=800 \mathrm{G}$. When searching for static solutions, we chose the following sample sizes: $L_{x}=80, \ldots, 140 \mathrm{~nm} ; L_{y}=400 \mathrm{~nm}$; and $L_{z}=750 \mathrm{~nm}$ (the distance between planes $\Lambda_{\mathrm{I}}$ and $\Lambda_{\mathrm{II}}$ was taken to be $L_{z} / 2$ ). To perform numerical minimization of energy (1), we used the method of conjugate gradients, which is based on the Fletcher-Reeves algorithm. To simulate the dynamic processes, we solved Eq. (5) with $\alpha=0.01$ using the fifth-order Runge-Kutta method in a sample with sizes $L_{x}=100 \mathrm{~nm}, L_{y}=400 \mathrm{~nm}$, and $L_{z}=750 \mathrm{~nm}$. The calculations were carried out with the OOMMF software package [3] using discretization on a rectangular mesh at a step of $5 \mathrm{~nm}$ along each coordinate (mesh step did not exceed absolute singledomain size $\left.l=\sqrt{A / 2 \pi} / M_{s}\right)$.

\section{RESULTS AND DISCUSSION}

\subsection{Static Configurations}

The results of energy minimization are presented in Table 1 as schematic sequences (notations) with the use of the pictograms introduced in Fig. 2. The symbol star indicates SP. Symbols X and Y correspond to "basic" TMSs, the structure of which will be described below. The sequence of the pictograms in a notation 


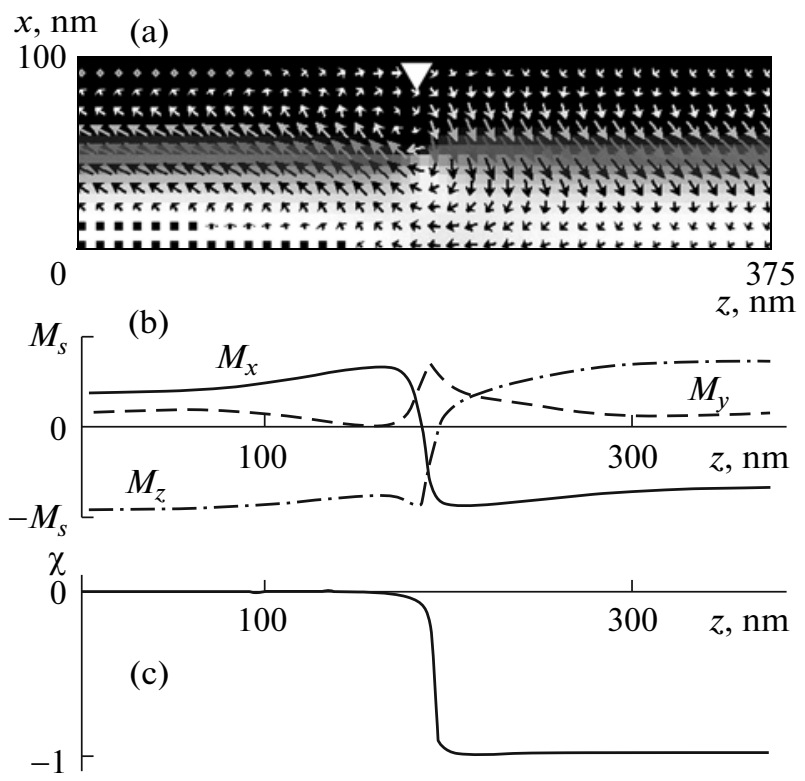

Fig. 4. Case A: TMS containing a singular (Bloch) point.

read from left to right reflects the order in which MS elements and domain wall regions are met during motion from $z=0$ to $z=L_{z}$ along a sample. MSs designated as $\mathrm{A}, \mathrm{B}$, and $\mathrm{C}$ differ from each other in the type of domain wall in the part of the sample located between planes $\Lambda_{\mathrm{I}}$ and $\Lambda_{\mathrm{II}}$ (see Fig. 2). Notations I and II correspond to TMSs localized near planes $\Lambda_{\mathrm{I}}$ and $\Lambda_{\mathrm{II}}$. We now consider the structures of various TMSs in detail.

TMS in case A separates left- and right-hand walls, which represent vortex tubes (with axes along $z$ ) in which the directions of $\mathbf{M}$ rotation in vortices are the same and the magnetization directions in the nearaxis regions are opposite. The magnetization configuration calculated for this case does not have poles at the film boundaries and consists of two SPs located in the vicinity of planes $\Lambda_{\mathrm{I}}$ and $\Lambda_{\mathrm{II}}$ at the sites of connection of the axes of vortex tubes. For a mesh approximation, SP represents a local region in which the angles between vectors $\mathbf{M}$ at neighboring nodes are not low. Figure 4a shows the section of the part of the sample confined by the planes $z=0$ and $z=375 \mathrm{~nm}$ that was made by the plane $y=200 \mathrm{~nm}$. Here, the white triangle indicates the position of SP, and the background color intensity is proportional to $M_{y}$ (higher value corresponds to lighter background). The one-dimensional distribution of $\mathbf{M}$ along the line $x=50 \mathrm{~nm}$ and $y=200 \mathrm{~nm}$ passing through SP has a singularity at the SP (Fig. 4b). Using Eq. (7), we numerically find the values of $\chi$ and detect a jump $\chi=0 \longrightarrow-1$ in the $\chi(\varsigma)$ curve when $\varsigma$ coincides with the $z$ coordinate of SP (Fig. 4c). The region of nonhomogeneity of $\mathbf{M}$ on the mesh that is caused by the presence of SP has finite sizes; as a result, the jump is smoothed.

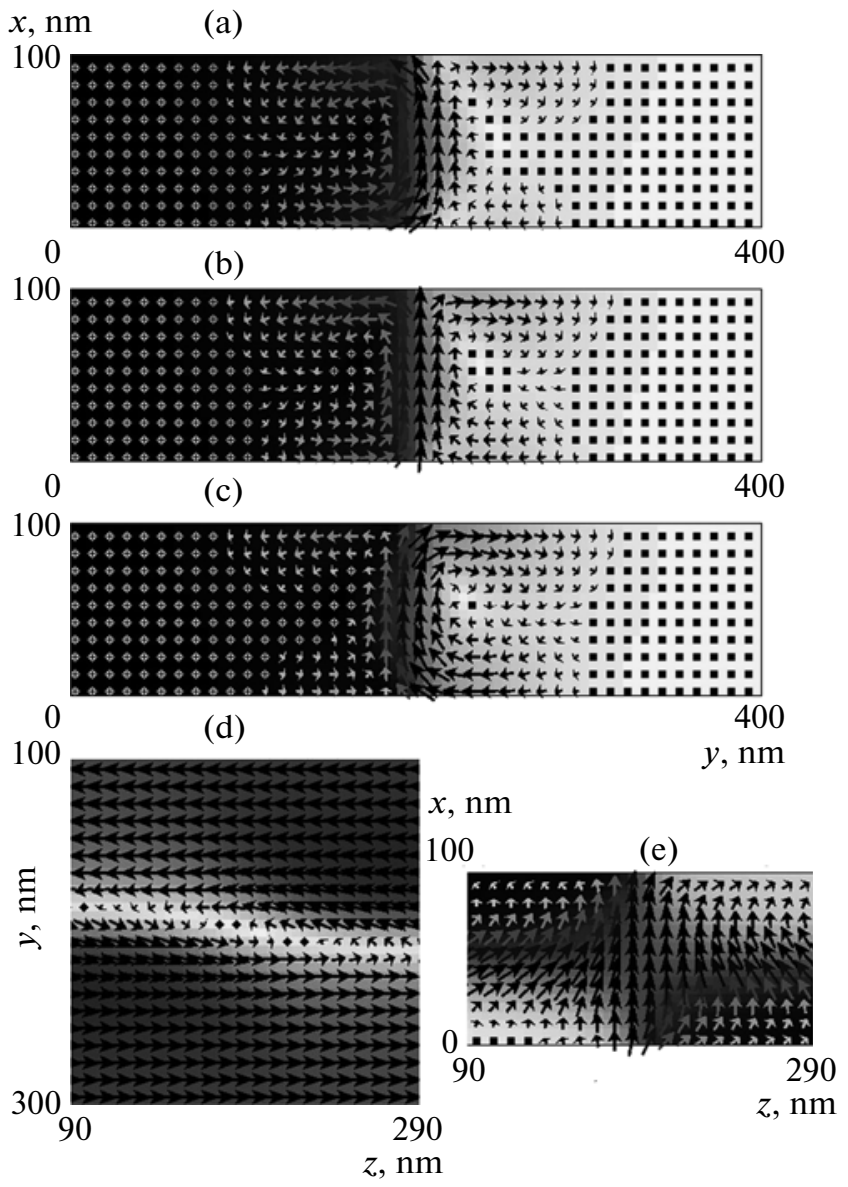

Fig. 5. Case B: M configuration in the sections of the sample containing X-type VBL by the planes (a) $z=160 \mathrm{~nm}$, (b) $z=180 \mathrm{~nm}$, (c) $z=200 \mathrm{~nm}$, (d) $x=50 \mathrm{~nm}$, and (e) $y=$ $200 \mathrm{~nm}$.

TMSs in cases B and C are the types of VBLs having different internal structures, and we will call them $\mathrm{X}$ - and Y-type VBLs (see Table 1).

$\mathrm{X}$-type VBL has a vortex and an antivortex on the opposite boundary surfaces; at their centers, the direction of $\mathbf{M}$ is the same and normal to these surfaces. If we go along axis $z$ and consider $\mathbf{M}$ in sections $z=$ const, we can find that the central surface of the wall $M_{z}(\mathbf{r})=0$ is flattened as TMS is approached. Here, the near-axial region of the vortex shifts toward the nearest domain (Fig. 5a) and coalesces with it; as a result, the vortex tube disappears. The disappearance of a vortex on one side of the central surface of the domain wall is accompanied by the appearance of a vortex on the other side: the near-axis region with the opposite value of $M_{z}$, which turns out to be surrounded by a new vortex tube, detaches from the second domain (Fig. 5c). A symmetric two-vortex structure forms in the section $z=180 \mathrm{~nm}$ passing through the center of TMS (Fig. 5b). The distribution of $\mathbf{M}$ in the section $y=$ $200 \mathrm{~nm}$ has a crosslike shape (Fig. 5e). The center of 


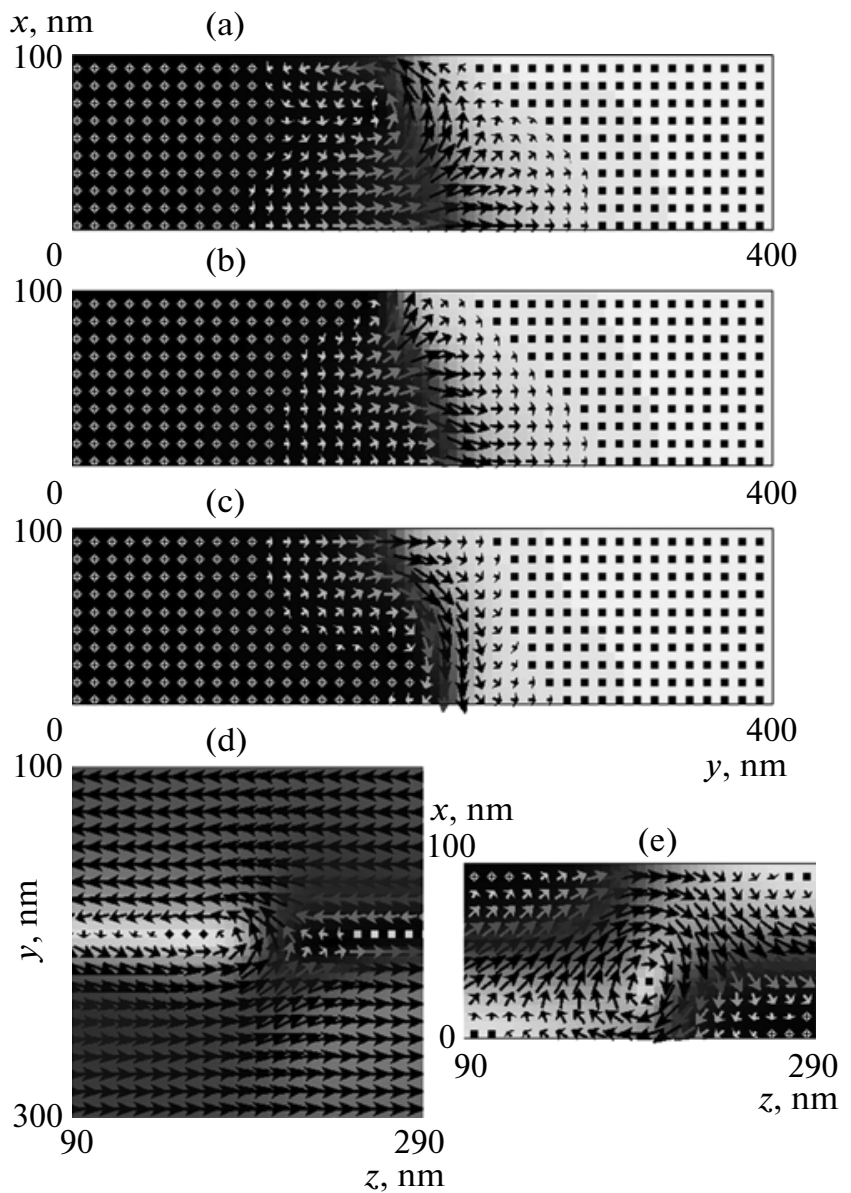

Fig. 6. Case $C$ : $M$ configuration in the sections of the sample containing Y-type VBL by the planes (a) $z=170 \mathrm{~nm}$, (b) $z=182 \mathrm{~nm}$, (c) $z=195 \mathrm{~nm}$, (d) $x=50 \mathrm{~nm}$, and (e) $y=$ $200 \mathrm{~nm}$.

the wall is closer to different domains on different sides of TMS (see Fig. 5d).

Y-type VBL has a more complex structure of $\mathbf{M}$ distribution. Now, at the centers of the vortex and antivortex that form on the opposite film boundary surfaces, vectors $\mathbf{M}$ are perpendicular to these surfaces and are directed in opposite directions. As follows from Figs. 6a and 6b, the vortex tube of the left-hand wall with counterclockwise rotation fails when the near-axis region of this tube reaches the upper film boundary. When going further in the positive direction along axis $z$, we see that a new near-axis region of the vortex tube, which belongs to left-hand wall with clockwise $\mathbf{M}$ rotation in the vortex, forms at the lower surface boundary (Fig. 6c). The Y-type VBL in the section created by the plane $y=200 \mathrm{~nm}$ has a transverse vortex structure (Fig. 6e). This structure creates the possibility of formation of poles with oppositely directed $\mathbf{M}$ vectors on the boundary film surfaces in the absence of a Bloch point between the poles. Y-type VBL has two subtypes, which differ in the magnetization vector direction at the center of the vortex shown

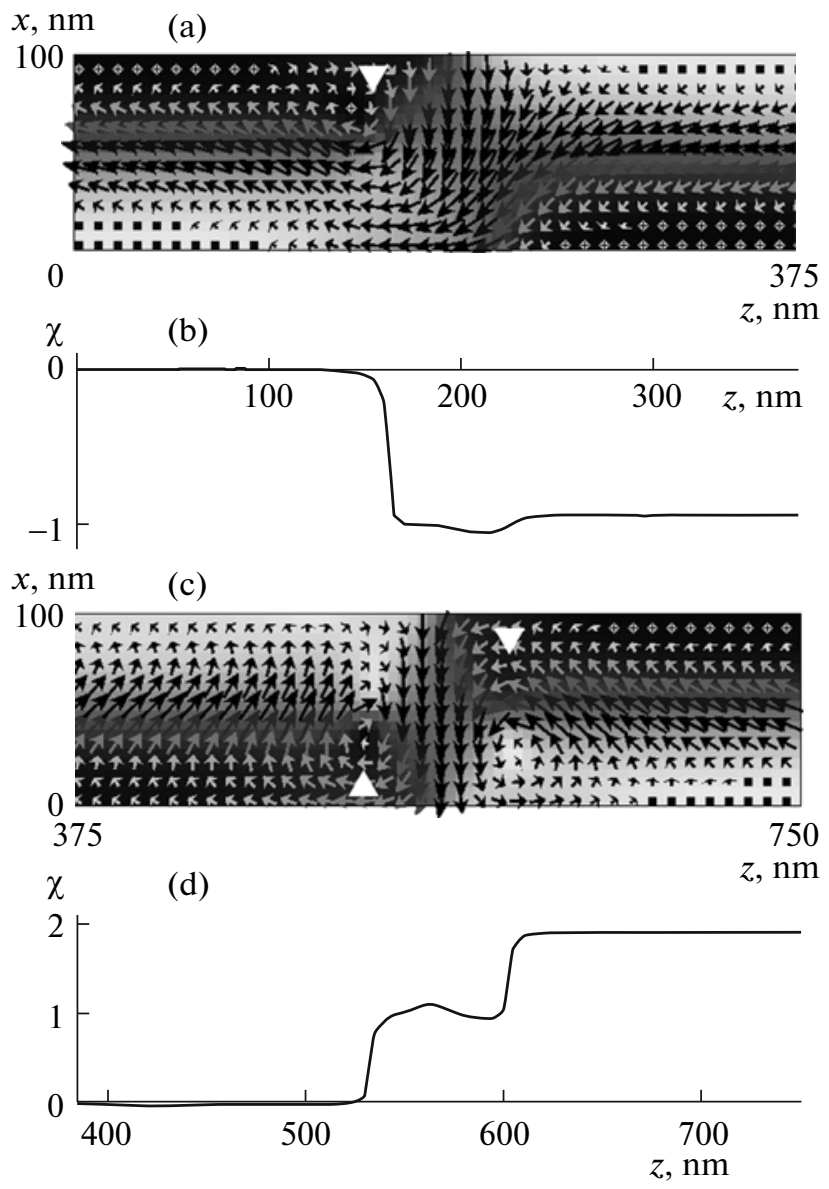

Fig. 7. Clusters $C_{2}(I)$ and $B_{1}(I I)$ consisting of X-type VBL and one or two singular points. The $\chi(\varsigma)$ curves in (b) and (d) correspond to the images in the sections created by the plane $y=200 \mathrm{~nm}$ in (a) and (c).

in Fig. 6e; in Table 1, these subtypes are designated as $\mathrm{Y}$ and $\overline{\mathrm{Y}}$.

Structures $\mathrm{B}_{1}(\mathrm{II}), \mathrm{C}_{1}(\mathrm{I}), \mathrm{C}_{2}(\mathrm{I}, \mathrm{II})$, and $\mathrm{C}_{4}(\mathrm{I}, \mathrm{II})$ are clusters consisting of X-type VBL and one or two SPs. Figures $7 \mathrm{a}$ and $7 \mathrm{c}$ show the section of the $\mathbf{M}$ distributions for structures $\mathrm{C}_{2}(\mathrm{I})$ and $\mathrm{B}_{1}(\mathrm{II})$ by the plane $y=$ $200 \mathrm{~nm}$, and the white triangles indicate the SP positions. The corresponding $\chi(\varsigma)$ curve is depicted under each section. It follows from these curves that $\chi=-1$ for SP entering into $\mathrm{C}_{2}$ (I) (Fig. 7b) and $\chi=1$ for each of the two SPs entering into $\mathrm{B}_{1}$ (II) (Fig. 7d). A nonmonotonic character of the $\chi(\varsigma)$ function at the site of VBL location can be explained by the errors of finitedifference approximation.

The stability of SP-containing clusters was tested as follows: the $\mathbf{M}$ fields describing them were summed with random field $\mathbf{M}_{r}\left(\left|\mathbf{M}_{r}\right| \sim M_{s} / 2\right)$, and the resulting fields were scaled so that the condition $|\mathbf{M}|=M_{s}$ to be met. Then, the energy is again minimized. As a result, we obtained magnetization distributions that were almost identical to the initial distributions. 
At film thicknesses $L_{x}<150 \mathrm{~nm}$, the interaction of TMSs located a distance $L_{z} / 2=375 \mathrm{~nm}$ is considered to be weak. It follows from Fig. 8a that the energy of a domain wall without TMS is minimal; that is, a wall with TMS of any type represents a metastable state. This result does not agree with the conclusions that are usually drawn when a magnetically uniaxial film with EA lying in its plane is considered in terms of the model of a one-dimensional magnetization distribution. This discrepancy is assumed to indicate the inadequacy of the one-dimensional model. From the standpoint of the one-dimensional approach, VBLs should be stable, since they decrease the poles on the boundary film surfaces in the stripes formed by the intersection of a domain wall with these surfaces. In more realistic models, which allow for the two-dimensional and three-dimensional character of $\mathbf{M}$ distributions, vortexlike objects with Néel regions can appear on the film surfaces. In this case, the magnetic flux is almost completely closed inside the film, which leads to the disappearance of the cause of energy gain due to the presence of VBLs.

As follows from experimental works, various types of TMSs exist (see, e.g., [9, 10, 32]). In particular, some fragments of the images of the surface magnetization distributions obtained in [32] with high-resolution Kerr microscopy are likely to correspond to TMSs of types A, B, and C (i.e., structures T3, T1, and T2 in terms of [32]). The appearance of TMS can be related to the history of domain structure formation. Moreover, VBLs can be created artificially, e.g., by the application of locally inhomogeneous pulsed magnetic fields to a film.

It is seen in Fig. 8b that the TMS energy per film thickness increases with the thickness. This fact indicates that TMSs are three-dimensional rather than linear objects, so that their changes cannot be reduced to simple elongation in thick films.

The partial energy densities shown for the particular case of X-type VBL in Fig. 8c are typical: approximately the same proportions between energies are characteristic of other TMSs. The main contribution is seen to be made by exchange energy. The contribution of magnetostatic energy turns out to be significantly smaller because of a well closed magnetic flux. The contribution of anisotropy energy is small because of the small quality factor of the films under study.

\subsection{Dynamic Solutions and Their Properties}

In Table 2, we present a qualitative description of the dynamic processes caused by the initial $\mathbf{M}$ configurations determined from static configurations (see Table 1) by moving TMSs toward each other (see Fig. 3). The dynamic restructuring of the $\mathbf{M}$ distribution occurs as the generation or annihilation of SPs and X- and Y-type VBLs. We indicate the initial distances between TMSs containing X-and Y-type VBLs
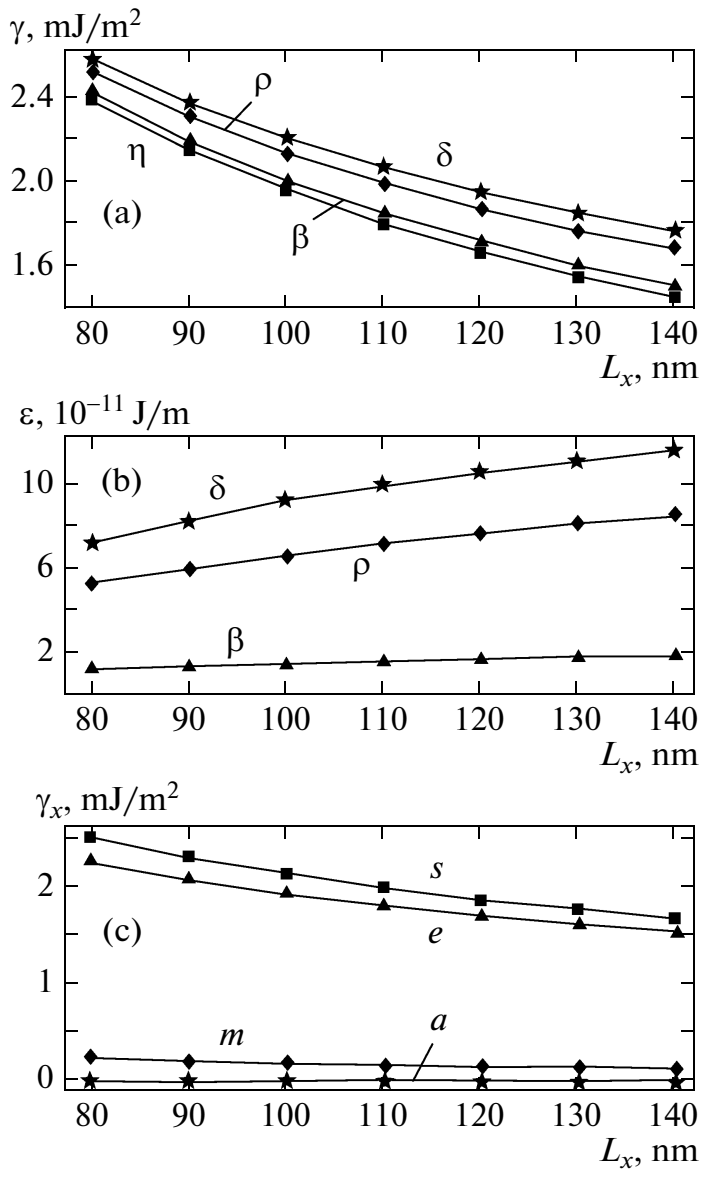

Fig. 8. Film-thickness dependences of (a) the surface energies of a wall without TMS $(\eta)$ or with two TMSs $(\beta$, $\rho, \delta$ ), (b) the TMS energies per film thickness, and (c) the surface partial energy densities for a film with two X-type VBLs. These data correspond to walls with SP $(\beta)$, X-type $\operatorname{VBL}(\rho)$, and Y-type VBL $(\delta)$. The family of curves in (c) consists of total energy $s$, exchange energy $e$, magnetostatic energy $m$, and anisotropy energy $a$.

as distances $d_{\bar{V} \bar{V}}$ between a vortex and an antivortex at the boundary $x=100 \mathrm{~nm}$. We now consider the dynamic processes in particular cases in more detail.

When calculating the $\chi(\varsigma)$ function, we can easily show that, in case A, we have two SPs, where $\chi=-1$ for the left SP and $\chi=1$ for the right SP. The dynamics simulation demonstrates that, when artificially placed at a distance of $50 \mathrm{~nm}$, SPs move away from each other. Figures $9 \mathrm{a}$ and $9 \mathrm{~b}$ depict the sections of SP-containing M distributions created by the plane $y=200 \mathrm{~nm}$. The rate of increase of distance $d$ between SPs decreases in time (Fig. 9c).

In case $\mathrm{B}$, two X-type VBLs annihilate, which results in the formation of a strongly excited wall without TMS. The inhomogeneities that appear due to piecewise-linear transformation lead to the formation of a low-amplitude diverging wave, which leaves the VBL localization region in about 60 ps. Then, two vortex-antivortex pairs located at different boundary film 
Table 2. Configurations of juxtaposed TMSs and qualitative description of dynamic processes

\begin{tabular}{|c|c|c|c|c|}
\hline \multirow{2}{*}{$\begin{array}{l}\text { Type } \\
\text { of MS }\end{array}$} & \multicolumn{2}{|c|}{ Juxtaposed TMSs } & \multirow{2}{*}{ Restructuring } & \multirow{2}{*}{ Result } \\
\hline & $\longrightarrow$ & $\longleftarrow$ & & \\
\hline A & & & No & $\begin{array}{l}\text { Two SPs moving away from } \\
\text { each other }\end{array}$ \\
\hline B & & & Annihilation of two X-type VBLs & Magnetization oscillations \\
\hline $\mathrm{C}$ & & & No & YY cluster \\
\hline $\mathrm{B}_{1}$ & & & No & $\mathrm{X} * \mathrm{X}$ cluster, moving away $\mathrm{SP}$ \\
\hline $\mathrm{C}_{1}$ & & & $\begin{array}{l}\text { Annihilation of X- and Y-type VBLs, } \\
\text { generation of SP }\end{array}$ & $\begin{array}{l}\text { Two moving SPs, magnetization } \\
\text { oscillations }\end{array}$ \\
\hline $\mathrm{C}_{2}$ & & & Annihilation of two X-type VBLs & $\begin{array}{l}\text { Two moving SPs, magnetization } \\
\text { oscillations }\end{array}$ \\
\hline $\mathrm{C}_{3}$ & & & $\begin{array}{l}\text { Annihilation of Y- and } \bar{Y} \text {-type VBLs, } \\
\text { generation and annihilation of two SPs }\end{array}$ & Magnetization oscillations \\
\hline
\end{tabular}

surfaces move toward each other and annihilate almost simultaneously. Figure 10 shows the section of the $\mathbf{M}$ distribution by the plane $y=200 \mathrm{~nm}$ at various times. The initial positions of VBLs $\left(d_{V \overline{\mathrm{V}}}=70 \mathrm{~nm}\right)$ are illustrated at the bottom $(t=0.03 \mathrm{~ns})$ and annihilation is shown below ( $t=0.44 \mathrm{~ns})$. The black arrows point to the direction of $\mathbf{M}$ vectors at the centers of the vortex and antivortex on the surface $x=0 \mathrm{~nm}$. The energy releasing during annihilation results in the formation of bending wall vibrations and spin waves. The waves move along the wall and cannot propagate deep into domains because of conditions (3). In the time interval from $t=0.8 \mathrm{~ns}$ to $t=1 \mathrm{~ns}$, there exists a single wave having two maxima indicated by black triangles and moving to the right.

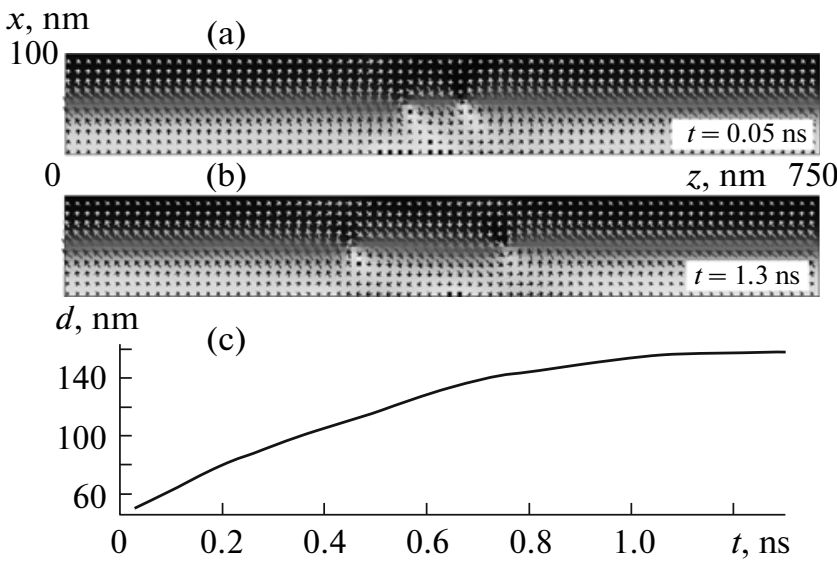

Fig. 9. Case A: singular (Bloch) points moving away from each other.
A similar dynamic picture is observed in case $\mathrm{C}_{2}$. The difference consists in the fact that the initial nonequilibrium distribution of $\mathbf{M}$ contains two SPs along with two X-type VBLs $\left(d_{\bar{v} \bar{v}}=70 \mathrm{~nm}\right)$. The dynamics simulation for about 1 ns demonstrates that the vibrations having appeared after the annihilation of vortexantivortex pairs and the disappearance of VBLs force SPs to shift; however, they do not annihilate.

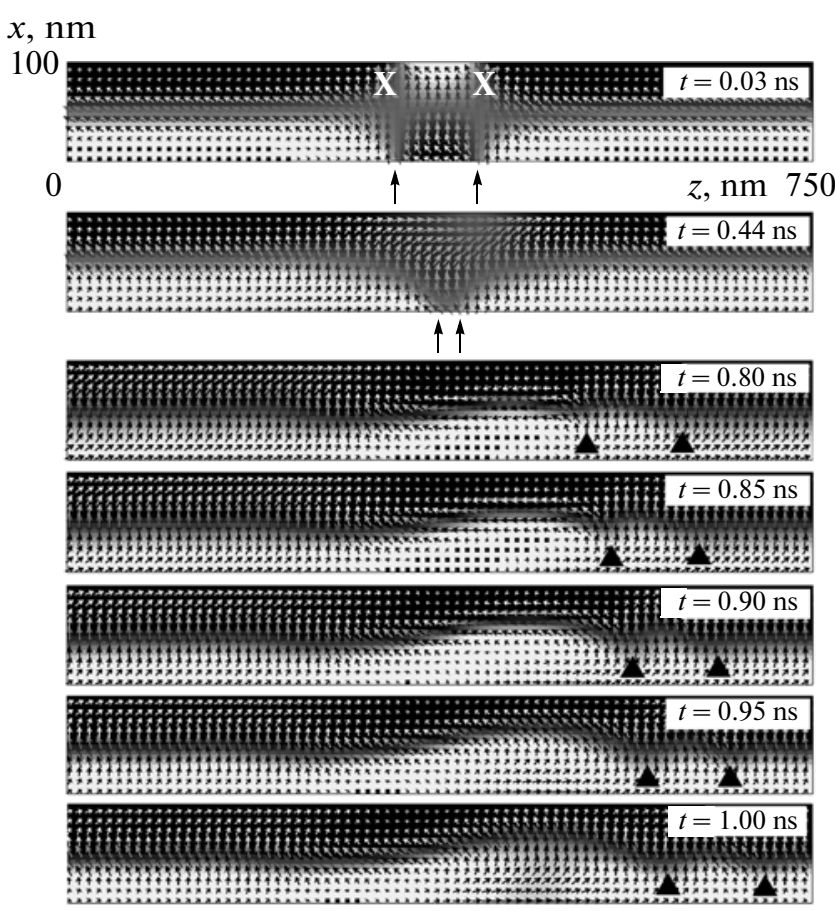

Fig. 10. Case B: annihilation of two X-type VBLs. 


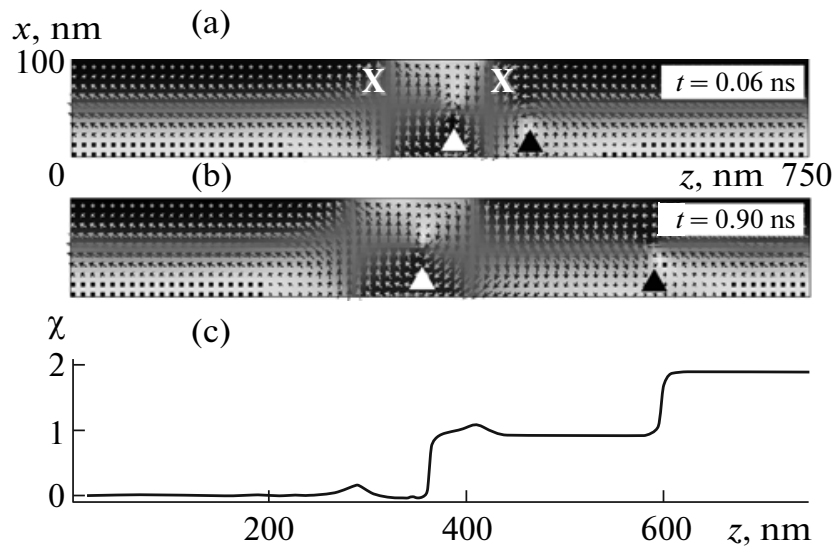

Fig. 11. Case $B_{1}$ : structure of two X-type VBLs and two SPs.

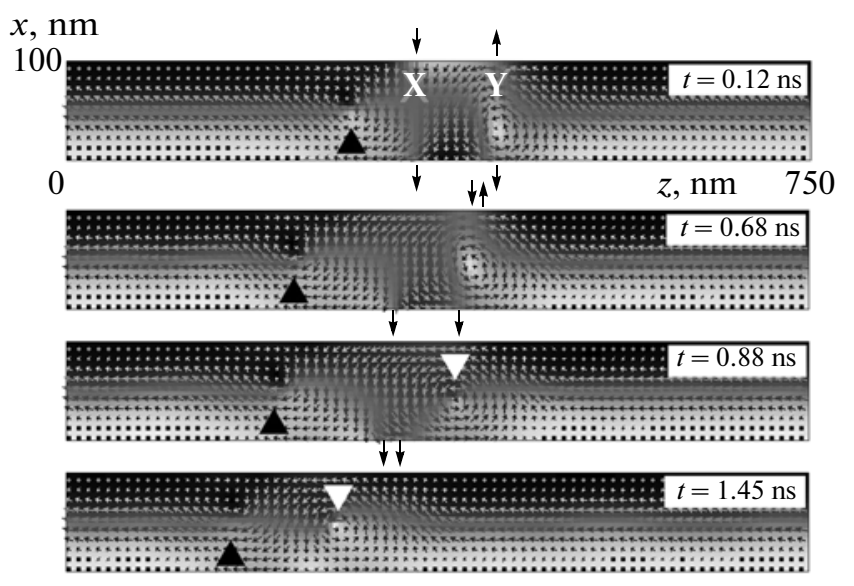

Fig. 12. Case $C_{1}$ : annihilation of $X$ - and Y-type VBLs accompanied by the generation of SP.
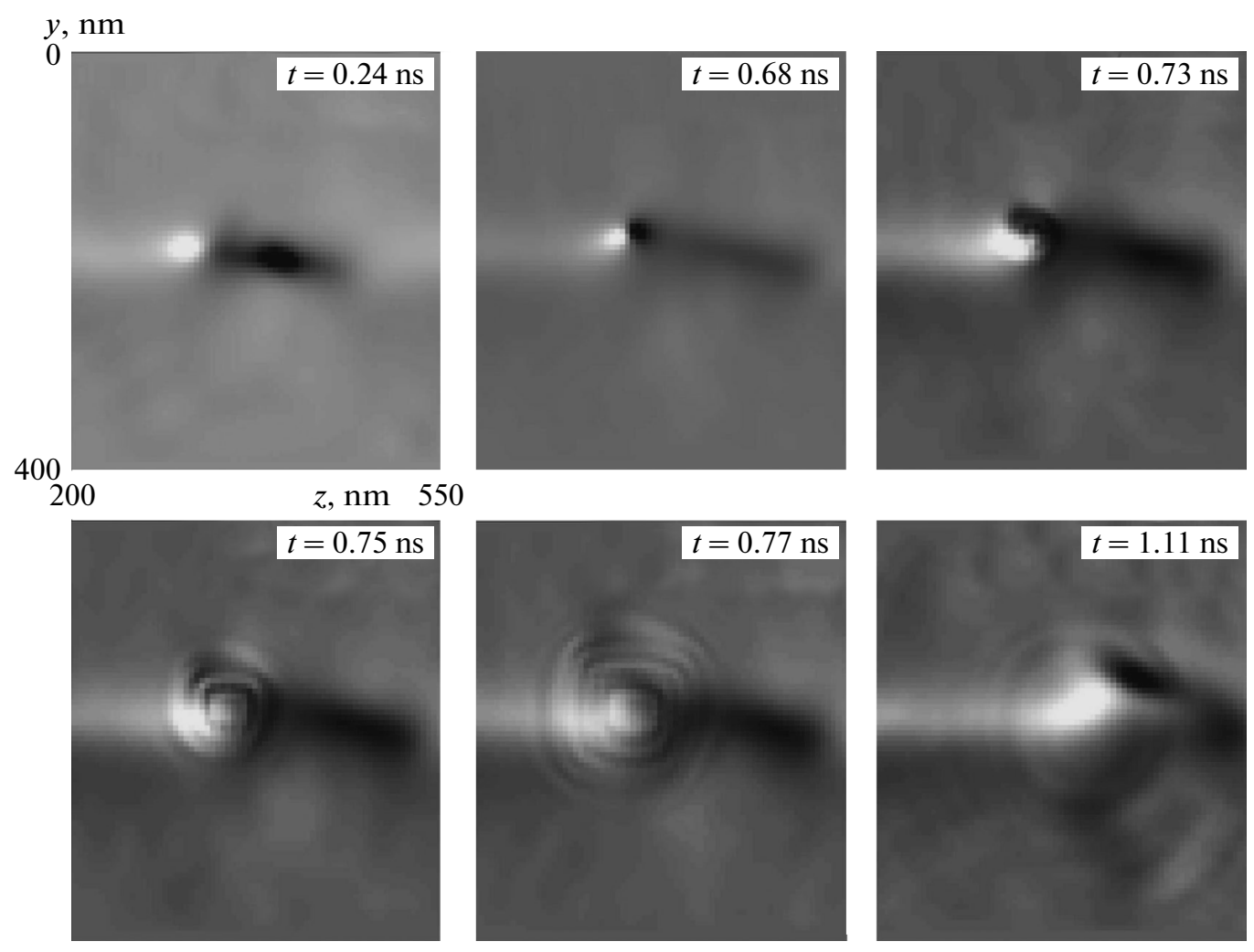

Fig. 13. Case $C_{1}$ : surface spin waves appearing during the annihilation of vortex-antivortex pairs.

In case $\mathrm{B}_{1}$, a structure consisting of two SPs and two X-type VBLs also forms after the approach of VBLs (Fig. 11, $d_{\bar{v} \bar{v}}=100 \mathrm{~nm}$ ). However the sequence of these elements in the $z$ direction is another: two VBLs are separated from each other by SP located between them. Simulation for about $1 \mathrm{~ns}$ showed that VBLs do not annihilate, only small dis- placements are observed in the $\mathrm{X} * \mathrm{X}$ structure, and the second SP moves rapidly to the right (in Figs. 11a and $11 \mathrm{~b}$, the SP positions are indicated by black and white triangles).

It is convenient to analyze the SP motion by plotting the $\chi(\varsigma)$ function at various times. The curve in Fig. 11c shows that the local field $\mathbf{M}$ inhomogeneities 

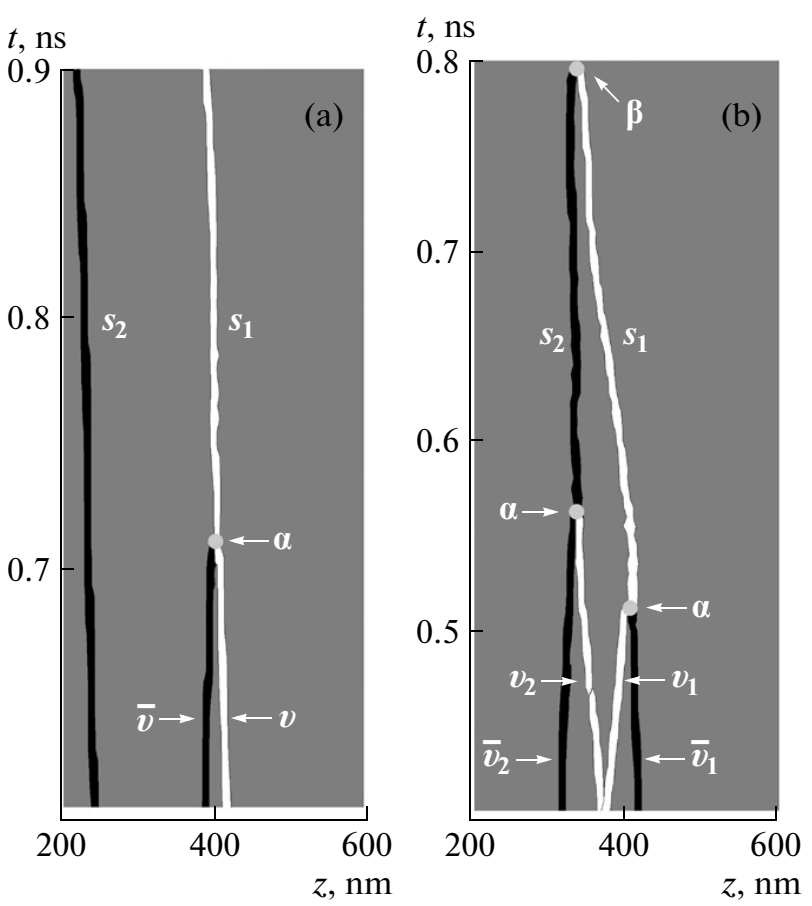

Fig. 14. Trajectories of vortices, antivortices, and SPs for cases (a) $\mathrm{C}_{1}$ and (b) $\mathrm{C}_{3}$.

observed visually do correspond to two SPs with $\chi=1$ (Figs. 11a, 11b). Another method of SP visualization, which is based on constructing $M_{x}$ and $M_{y}$ isosurfaces, is described in [24].

As noted in [24], SP forms in 10-nm-thick permalloy films during the annihilation of a vortex and an antivortex that have antiparallel magnetization vectors at their centers. We will show that this behavior also takes place when a vortex and an antivortex entering into TMS annihilate. We consider the annihilation of $\mathrm{X}$ - and Y-type VBLs (case $\mathrm{C}_{1}$ ). In Fig. 12 (which illustrates the mains stages of MS evolution), the black arrows indicate the magnetization vector directions at the centers of the vortices and the black triangle indicates SP. A vortex and an antivortex located at a distance $d_{\bar{V} \bar{V}}=100 \mathrm{~nm}$ from each other on the plane $x=$ $100 \mathrm{~nm}$ move toward each other and annihilate at $t=$ $0.68 \mathrm{~ns}$, generating the second SP (indicated by a white triangle). Then, at $t=0.88 \mathrm{~ns}$, the vortex and antivortex annihilate on the surface $x=0$ without SP formation.

The annihilation of a vortex and an antivortex leads to energy release and the appearance of a wave process both on the surface and in the volume of the film. In Fig. 13, the gray tone intensity is proportional to the $x$ component of $\mathbf{M}$ on the surface $x=100 \mathrm{~nm}$ (case $\mathrm{C}_{1}$ ). We observe the approaching of a vortex and an antivortex at $t<0.68 \mathrm{~ns}$ and various stages of the propagation of the wave induced by annihilation at the surface $x=$ $100 \mathrm{~nm}$ in the time interval $t=0.68-0.77 \mathrm{~ns}$. At $t=$
$1.11 \mathrm{~ns}$, the wave generated by the annihilation of the second vortex-antivortex pair on the surface $x=0 \mathrm{~nm}$ reaches the surface $x=100 \mathrm{~nm}$.

It is convenient to analyze the motion and evolution of vortices, antivortices, and SPs using functions $d j(\varsigma, t) / d \varsigma$ and $d \chi(\varsigma, t) / d \varsigma$, the values of which can be found using Eqs. (6) and (7) and the data of $\mathbf{M}$ configurations at various times. The trajectories of vortices, antivortices, and SPs, which are made of deltalike bursts at the sites of the steps in the $j(\varsigma, t)$ and $\chi(\varsigma, t)$ functions, are visible in the halftone images in Fig. 14. The dark and bright lines indicate the MS trajectories characterized by topological invariants $(j$ for vortices and antivortices and $\chi$ for SPs) of -1 and +1 , respectively.

For case $\mathrm{C}_{1}$, the trajectories of motion of various MSs are shown in Fig. 14a. The dark and bright lines below point $\alpha$ are the trajectories of vortex $v$ and antivortex $\bar{V}$ that belong to X-type VBL and Y-type VBL, respectively, and are located on the boundary surface $x=100 \mathrm{~nm}$. The annihilation of these MSs occurring at point $\alpha$ results in the generation of SP $s_{1}$. The trajectory of another SP $\left(s_{2}\right)$ is shown on the left in this figure.

In case $\mathrm{C}_{3}$, the approaching of $\mathrm{Y}$ - and $\overline{\mathrm{Y}}$-type $\operatorname{VBLs}\left(d_{\bar{V} \bar{V}}=85 \mathrm{~nm}\right)$ leads to nonsimultaneous annihilation of two vortex-antivortex pairs: vortex $v_{1}$ and antivortex $\bar{v}_{1}$ first annihilate at the boundary $x=0$, and vortex $v_{2}$ and antivortex $\bar{v}_{2}$ then annihilate at the boundary $x=100 \mathrm{~nm}$ (Fig. 14b). As a result, SPs $s_{1}$ and $s_{2}$ with different values of $\chi$ appear at points $\alpha$. In a certain time, these SPs reach each other and annihilate at point $\beta$.

We now characterize the dynamic processes that occur when TMSs move toward each other from the standpoint of topological transformations. In cases A, $\mathrm{C}$, and $\mathrm{B}_{1}$, transformations of this type are absent. In cases $\mathrm{B}$ and $\mathrm{C}_{2}$, there exist surface transformations caused by the annihilation of vortex-antivortex pairs at the film boundaries. Finally, in cases $\mathrm{C}_{1}$ and $\mathrm{C}_{3}$ (Fig. 14), the chains of topological transformations include both surface transformations (interaction of vortices and antivortices at the film boundaries) and solid transformations (generation and annihilation of SPs).

\section{CONCLUSIONS}

Based on the numerical simulation that exactly takes into account all basic interactions in magnetically uniaxial films with in-plane anisotropy (including dipole-dipole interaction), we found the micromagnetic structures with a three-dimensional magne- 
tization $\mathbf{M}$ distribution that separate the regions with different vortex arrangements and (or) different directions of magnetization rotation in the vortices in asymmetric vortexlike domain walls.

It was shown that three basis structures, namely, SP (Bloch point), X-type VBL, and Y-type VBL, correspond to the three possible types of transitions between the regions of various types in domain walls. The last two structures are characterized by the presence of a vortex on one film surface and an antivortex on the other surface. The magnetizations at the centers of a vortex and an antivortex are normal to the film surfaces and are either codirectional (type $\mathrm{X}$ ) or antidirectional (type $\mathrm{Y}$ ). The three-dimensional structures of VBL with one vortex (antivortex) at each film boundary, single SP, and clusters consisting of VBL and SP were completely described. Y-type VBL was shown to have a vortex structure in the plane normal to the film surface and parallel to EA. We also found two Y-type VBL modifications, which differ in the $\mathbf{M}$ direction at the center of a vortex.

Based on the obtained numerical data, we found topological invariants for single SPs and the SPs entering into clusters; as a result, we were able to perform topological classification of TMSs.

The asymmetric domain walls containing VBLs, SPs, and clusters were shown to be metastable. Nevertheless, being topological structures, they are rather stable, and energy is required to destroy them. However, the only stable object is represented by an asymmetric vortex-free Bloch wall.

The total energy of asymmetric walls with any TMS was found to decrease with increasing film thickness, which is mainly related to the decrease in the exchange energy caused by a decrease in the magnetization inhomogeneity in thick films.

We found the partial contributions of the energies of various types to the total energy of asymmetric Bloch walls with TMS and showed that the main contribution to the total energy is related to a nonuniform exchange interaction, as in the case of TMS-free walls.

We also studied model configurations in which TMSs were artificially located at small distances for them to interact with each other and found various scenarios of the dynamic behavior of these systems. They are as follows:

(a) time-independent configurations;

(b) motion without restructuring (motion of two SPs far away from each other);

(c) complete annihilation of TMS, which is accompanied by energy release and the retention of a vibrating asymmetric wall of a certain type (annihilation of two X-type VBLs);

(d) partial annihilation of TMS, which is accompanied by energy release and the retention of domain wall regions of different types separated by remaining
TMSs (annihilation of two X-type VBLs with the retention of two SPs);

(e) partial or complete annihilation of TMS, which is accompanied by the appearance of new TMSs (annihilation of X- and Y-type VBLs and the generation of SP, annihilation of $\mathrm{Y}$ - and $\bar{Y}$-type VBLs and the generation of two SPs followed by their annihilation).

We developed a method to analyze SP-containing micromagnetic structures, which is based on the calculation of the topological invariants of regions having a variable boundary position.

\section{ACKNOWLEDGMENTS}

This work was supported in part by the Russian Foundation for Basic Research, project no. 11-0200931.

\section{REFERENCES}

1. L. Landau and E. Lifshitz, Phys. Z. Sowjetunion 8, 153 (1935).

2. W. F. Brown, Micromagnetics (Interscience, New York, 1963; Nauka, Moscow, 1979).

3. M. J. Donahue and D. G. Porter, OOMMF User's Guide, Version 1.0, NISTIR 6376 (National Institute of Standards and Technology, Gaithersburg, Maryland, United States, 1999).

4. S. A. Konishi, IEEE Trans. Magn. 40, 1838 (1983).

5. S. S. P. Parkin, M. Hayashi, and L. Thomas, Science (Washington) 320, 190 (2008).

6. A. Malozemoff and J. Slonczewski, Magnetic Domain Walls in Bubble Materials (Academic, New York, 1979; Mir, Moscow, 1982).

7. A. E. Labonte, J. Appl. Phys. 40, 2450 (1969).

8. J. N. Chapman, G. R. Morrison, J. P. Jacubovics, and R. A. Taylor, J. Magn. Magn. Mater. 49, 277 (1985).

9. B. E. Argyle, B. Petek, M. E. Re, F. Suits, and D. A. Herman, J. Appl. Phys. 63, 4033 (1988).

10. C. G. Harrison and K. D. Leaver, Phys. Status Solidi A 15, 415 (1973).

11. S. Huo, J. E. L. Bishop, J. W. Tucker, W. M. Rainforth, and H. A. Davies, J. Magn. Magn. Mater. 177-181, 229 (1998).

12. S. Huo, J. E. L. Bishop, J. W. Tucker, W. M. Rainforth, and H. A. Davies, J. Magn. Magn. Mater. 218, 103 (2000).

13. M. Redjdal, A. Kakay, T. Trunk, M. F. Ruane, and F. B. Humphrey, J. Appl. Phys. 89, 7609 (2001).

14. M. Redjdal, A. Kakay, M. F. Ruane, and F. B. Humphrey, J. Appl. Phys. 91, 8278 (2002).

15. W. Döring, J. Appl. Phys. 39, 1006 (1968).

16. S. W. Yuan and H. N. Bertram, Phys. Rev. B: Condens. Matter 44, 12395 (1991). 
17. B. N. Filippov, L. G. Korzunin, and F. A. Kassan-Ogly, Phys. Rev. B: Condens. Matter 64, 104412 (2001).

18. B. N. Filippov, Low Temp. Phys. 28 (10), 707 (2002).

19. Ki-Suk Lee, Byoung-Woo Kang, Young-Sang Yu, and Sang-Koog Kim, Appl. Phys. Lett. 85, 1568 (2004).

20. Q. F. Xiao, J. Rudge, B. C. Choi, Y. K. Hong, and G. Donohoe, Appl. Phys. Lett. 89, 262507 (2006).

21. Ki-Suk Lee, K. Y. Guslienko, Jun-Young Lee, and Sang-Koog Kim, Phys. Rev. B: Condens. Matter 76, 174410 (2007).

22. R. Hertel, S. Gliga, M. Fahnle, and C. M. Schneider, Phys. Rev. Lett. 98, 117201 (2007).

23. Ki-Suk Lee, Sangkook Choi, and Sang-Koog Kim, Appl. Phys. Lett. 87, 192502 (2005).

24. R. Hertel and C. M. Schneider, Phys. Rev. Lett. 97, 177202 (2006).

25. Sangkook Choi, Ki-Suk Lee, K. Y. Guslienko, and Sang-Koog Kim, Phys. Rev. Lett. 98, 087205 (2007).
26. K. M. Lebecki, M. J. Donahue, and M. W. Gutowski, J. Phys. D: Appl. Phys. 41, 175005 (2008).

27. G. E. Volovik and V. P. Mineev, Sov. Phys. JETP 45 (6), 1186 (1977).

28. B. A. Dubrovin, S. P. Novikov, and A. T. Fomenko, Modern Geometry: Methods and Applications (Nauka, Moscow, 1979; Springer-Verlag, New York, 1991).

29. A. A. Thiele, Phys. Rev. Lett. 30, 230 (1973).

30. A. M. Kosevich, B. A. Ivanov, and A. S. Kovalev, Nonlinear Waves of Magnetization: Dynamical and Topological Solitons (Naukova Dumka, Kiev, 1983) [in Russian].

31. A. A. Belavin and A. M. Polyakov, JETP Lett. 22 (10), 245 (1975).

32. R. Schäfer, W. K. Ho, J. Yamasaki, A. Hubert, and F. Humphrey, IEEE Trans. Magn. 27, 3678 (1991).

Translated by K. Shakhlevich 